\title{
The Intersection Between AI and IP: Conflict or Complementarity?
}

\author{
Céline Castets-Renard
}

Published online: 21 January 2020

(C) Max Planck Institute for Innovation and Competition, Munich 2020

Artificial intelligence (AI) is everywhere. If Alan Turing raised the question of providing machines with a form of intelligence as early as $1950,{ }^{1}$ AI has since revealed its potential thanks to big data and the improvement of computing power. The term "artificial intelligence" was popularized by John McCarthy and Marvin Lee Minsky, organizers of the 1956 Dartmouth conference that made AI a field of research in its own right. AI refers to systems that demonstrate intelligent behavior by analyzing their environment and taking action, with a degree of autonomy, to achieve specific goals. "Intelligence" then refers to the fact that the machine imitates the cognitive functions associated with the human or animal brain, i.e. the ability to learn and solve problems. This may involve thinking or acting by imitating human behavior (cognitive approach) or rationality (computational approach). ${ }^{3}$ If, for several decades, AI experienced waves of enthusiasm and setbacks ("winters" of $\mathrm{AI}$ ), it is now part of everyday life, whether it is using a personal virtual or virtual assistant, or travelling in a semi-autonomous vehicle. It is also generating a craze for solving or anticipating complex problems in medicine, policing or justice among others. The adaptability of AIs greatly increases their applicable uses, thus different objectives can be pursued: automatic natural language processing, knowledge representation, automated reasoning, machine learning, computer vision and robotics to name a few. In this last case, AI systems are not only working on the software level, but they are also acting in a virtual world integrated into hardware.

\footnotetext{
1 A. M. Turing, "Computing Machinery and Intelligence", Mind, Volume LIX, Issue 236, Oct. 1950, p. $433-460$.

2 Artificial Intelligence: A European Perspective, Joint Research Centre, EUR 29425 EN, 2018.

3 S. Russell and P. Norvig, Artificial Intelligence: a Modern Approach, Pearson, 3rd edn., 2016 (introduction).
}

C. Castets-Renard $(\bowtie)$

Professor, Civil Law Faculty, University of Ottawa, Chair Holder Law, Accoutability, and Social Trust in AI, ANITI, ANR-3IA (France), Ottawa, Canada

e-mail: ccastets@uottawa.ca 
In this context, it comes as no surprise that this new technology encounters intellectual property in various ways. There are three main overlaps between AI and IP: AI as a technology that may assist in the management of intellectual property rights, intellectual property as a regime for the protection of AI, and IP as an obstacle to the transparency of AI systems. As one can see, the relationship between both of these is reciprocal: IP influences AI and AI influences IP. Moreover, the overlap between both can be beneficial as well as conflictual.

Firstly, AI is a helpful technology to manage intellectual property rights. AI in IP administration is being increasingly deployed in the administration of applications for IP protection. For instance, WIPO Translate and WIPO Brand Image Search use AI-based applications for automated translation and image recognition. Around the world, several IP offices have developed and deployed various other AI applications. In May 2018, a meeting was organized by WIPO to discuss these AI applications, encourage their sharing and the exchange of information. ${ }^{4}$

Secondly, intellectual property is a legal system that can protect AI. AI is already having, and is likely to have, a significant impact on the creation, production and distribution of economic and cultural goods and services. One of the main goals of IP policy is to stimulate innovation and creativity in the economic and technological areas, where AI and IP intersect easily. Different rights of IP laws could protect AI. At the same time, WIPO was encouraged by its member states to collate the significant government instruments of relevance to AI to standards. Patent and copyright codes are the most relevant systems of protection regarding AI, especially when inventions can be autonomously generated by AI. There are several reported cases of applications for patent protection in which the applicant has named an AI application as the inventor. Should the law permit that the AI application be named as the inventor, or should it be required that a human being is named as the inventor? If so, should the law give indications of the way to determine the human inventor or let the stakeholders take this decision by private arrangements? Moreover, the main question is who should be recorded as the owner of a patent involving an AI application? Are current legal provisions sufficient to consider the specificities of inventions generated by AI or should specific legal provisions be introduced? On the other hand, should the law exclude the availability of patent protection of autonomously generated inventions by an AI application? Furthermore, one can raise the question of the interpretation of patentability, namely inventive step or non-obviousness. In particular, what art does the standard refer to? Finally, the condition of disclosure could be challenging for an invention generated by an AI application. Specifically, how can the condition of disclosure be fulfilled where the algorithms of machine learning change over time? Should the data used to train an algorithm be disclosed or described in the patent application? Depending on the answers given to these questions, the lawmaker may be led to consider that a sui generis system of IP rights for AI-generated inventions should be raised to adjust innovation incentives for AI.

\footnotetext{
4 See WIPO, Conversation on Intellectual Property (IP) and Artificial Intelligence (AI): Draft Issues Paper on Intellectual Property Policy and Artificial Intelligence, WIPO IP/AI/2/GE/20/1, 13 December 2019.
} 
Similar questions could be raised regarding the copyright regime. While AI applications are capable of producing literary and artistic works autonomously, this capacity does not fit with the copyright system which is intimately associated with the human creative spirit and with respect and reward for the expression of human creativity. The main question raised here is whether the copyright should be attributed to original literary and artistic works that are autonomously generated by AI or should a human creator be required?

Thirdly, if one considers that AI systems should be protected by IP rights, this raises another issue that IP rights could yet be an obstacle to the transparency of AI systems. While there is an advocacy today for more transparency and accountability of algorithmic decision-making systems, the question remains of how to satisfy this requirement in cases where a machine learning process involves multiple data sources, dynamic development, and elements that are opaque, whether for technological or legal reasons? ${ }^{5}$ IP rights and trade secrets could create some barriers, and raise a conflict of norms between IP protection and the social need for transparency and accountability. Nevertheless, the disclosure for satisfying these goals does not concern the algorithmic rules themselves but only their results namely explanation as a result of their application. In other words, there is no risk of breaching IP rights and trade secrets. Moreover, such rights should not be used to avoid the disclosure and explanation of AI systems. This issue of transparency illustrates a case where the overlap between AI and IP is the most conflictual.

The questions raised above boil down to the overlap of AI and IP as important and interesting observations and points that have not yet been asked and therefore addressed. Here, issues have been presented, some of which should encourage scholars to find the balance between AI and IP.

Publisher's Note Springer Nature remains neutral with regard to jurisdictional claims in published maps and institutional affiliations.

\footnotetext{
5 See R. Wexler, "Life, Liberty, and Trade Secrets: Intellectual Property in the Criminal Justice System", 70 Stan. L. Rev. pp. 1343, 1373-74 (2018).
} 